\title{
Investigation of Spray Cooling Performance on Different Heat Transfer Surfaces
}

\author{
Jin Shicheng \\ School of Aviation Science and Engineering \\ Beijing University of Aeronautics and Astronautics \\ Beijing, China \\ 1670130062@qq.com \\ Zhang Yu \\ School of Aviation Science and Engineering \\ Beijing University of Aeronautics and Astronautics \\ Beijing, China \\ zhangyu_0601@163.com \\ Ji Yibin \\ Aviation Key Laboratory of Science and Technology on \\ Aero Electromechanical System Integration \\ Nanjing Engineering Institute of Aircraft Systems \\ Nanjing, China \\ jyb81121@163.com
}

\begin{abstract}
-with the rapid development of laser technology and high integrated circuit technology in the field of aerospace, the equipment thermal dissipation is increasing sharply. The traditional thermal control methods such as air natural convection, forced convection and heat pipe cooling cannot meet the demand of high heat flux rejection. Additionally, high heat flux cooling techniques are more difficult to apply to airborne weapons and electronic devices. Consequently, a new efficient cooling technology is urgently required for their thermal management. Because of the advantages such as reduced coolant flow rate, no delay boiling, low temperature difference between the working fluid and the heated surface, and high heat flux removal capability, spray cooling has been one of the most attractive method to solve the high power dissipation problem. For the special requirements of airborne laser weapons and high power electronics, experimental studies were conducted to investigate the heat transfer performance of spray cooling on different heated surfaces.
\end{abstract}

Keywords-component; spray cooling; airborne electronic equipment; green laser flow visualization; experimental study; heated surface structure

\section{INTRODUCTION}

With the rapid development of industrial technology, the electronic components become more and more miniature and compositive. The heat dissipation of the electronic devices is increasing. For instance, the largescale electronic equipment can generate waste heat up to $100-200 \mathrm{~W} / \mathrm{cm}^{2}$. Therefore, the energy consumption and heat dissipation problems are very serious ${ }^{[1 \sim 2]}$.

Under the action of high pressure gas or its own pressure, liquid is atomized to a mist of droplets whose diameter is about 20 to $100 \mu \mathrm{m}$. Some of the droplets impact the heated surface and come into being a liquid film. In the spray cooling process, the droplets collide

\author{
Pang Liping \\ School of Aviation Science and Engineering \\ Beijing University of Aeronautics and Astronautics \\ Beijing, China \\ pangliping@buaa.edu.cn \\ Yu Wenxuan \\ School of Aviation Science and Engineering \\ Beijing University of Aeronautics and Astronautics \\ Beijing, China \\ 542497747@qq.com
}

with the heated surface and cool it by a few means such as convection between the liquid film and heated surface, phase change from liquid to vapor and transient conduction when liquid contact with the surface. The heated liquid can be collected and recycled after it is cooled and cleaned. Therefore, a closed loop system is workable ${ }^{[3 \sim 5]}$.

Nowadays spray cooling has been widely applied in many fields. There has been spray cooling system used in military platforms in USA. Besides, spray cooling can be applied to the cooling of electronics components ${ }^{[6 \sim 8]}$.

\section{THE EXPERIMENTAL STUDY METHOD FOR SPRAY COOLING}

\section{A. Experimental apparatus}

Fig. 1 shows the flow chart of spray cooling system and Fig. 2 shows the overall experimental setup.

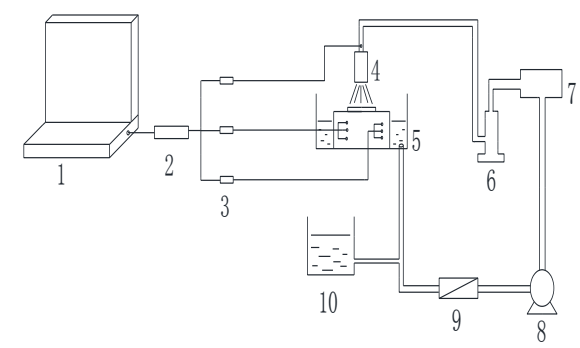

1 Computer; 2 Connector; 3 Data acquisition module; 4 Nozzle; 5 Collection sink; 6 Flow meter; 7 Pressure buffer; 8 Pump; 9 filter; 10 water reservoir

Figure 1. Simulation of high power electronic equipment 


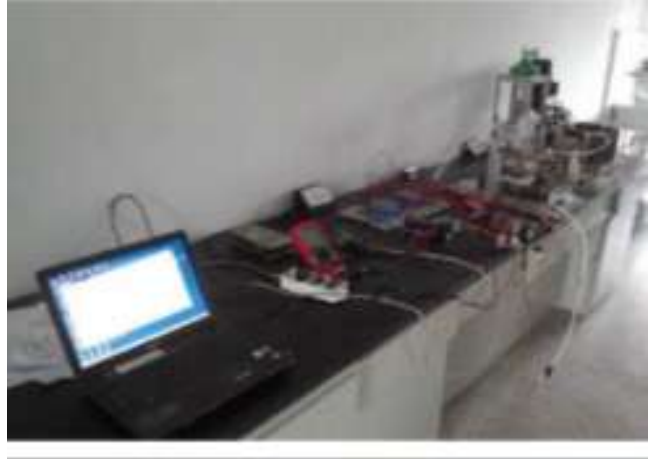

Figure 2. The overall experimental setup

The major components are described below.

The mechanical diaphragm metering pump provided by MNI corp has a rated voltage of $380 \mathrm{~V}$, a maximum pressure of $1.03 \mathrm{MPa}$ and maximum volume flow rate of $22 \mathrm{~L} / \mathrm{h}$.

1) The pressure buffer is used to keep the system pressure stable and reduce the pressure fluctuation caused by the pump. With it, the system pressure stabilize around $1.0 \mathrm{Mpa}$.

2) The check valve is closed at the beginning of the experiment and opened when the preset system pressure is reached.

3) The safety valve has a trigger pressure of $1.0 \mathrm{Mpa}$ and it will open once the system pressure is larger than that trigger pressure.

The pressure sensor and temperature thermocouples are used to observe the pressure and temperature of the measuring points every two second.

4) The rotor flowmeter is used to measure the liquid volume flow rate and its measurement range is $1-3 \mathrm{~L} / \mathrm{h}$.

5) Some data acquisition modules are used to collect the measurements of pressure, temperature and heat flux density. One power control module is used to set the heating power.

\section{B. Simulation of the heat dissipation from high power electronics}

In this experiment, a copper block and three heating rods are used to simulate the heat dissipation. The top surface is used for the heat transfer. The structure is shown in Fig. 3 and the simulation result is also shown in this picture.
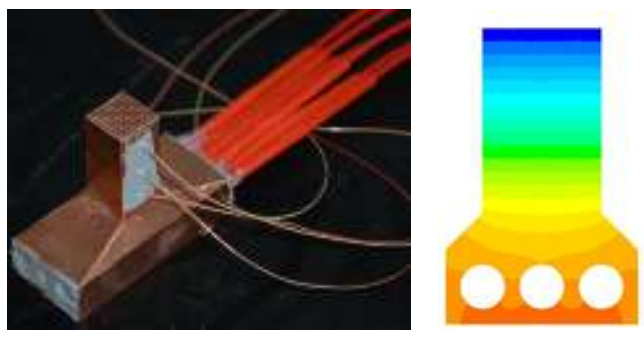

Figure 3. Structure of simulation heat source

Cartridge heaters are $6 \mathrm{~mm}$ in diameter, $60 \mathrm{~mm}$ in length, $167 \Omega$ in resistance and their total heating power is $360 \mathrm{~W}$. The copper block has a thermal conductivity of
$398 \mathrm{~W} /(\mathrm{m} \cdot \mathrm{K})$ at room temperature. Glass wool is used to reduce heat loss and its thermal conductivity is about 0.08 $\mathrm{W} /(\mathrm{m} \cdot \mathrm{K})$. Six K-type thermocouples have an accuracy of $\pm 1.5{ }^{\circ} \mathrm{C}$ and a range of -200 to $300{ }^{\circ} \mathrm{C}$. Stainless steel shell is used to separate the liquid with the heater.

Heater rods are linked up in parallel and placed at the bottom of the copper block. The power output for each heater rod can be regulated from 0 to $120 \mathrm{~W}$. In other words, the maximum output power of the heater assembly is $360 \mathrm{~W}$. The insulation material is used to fill the gap between the copper block and the stainless steel shell. Additionally, 704 adhesive is used to prevent the contact between the water and heater, and reduce the heat loss as well. The heat is transferred from the heater rods to the top surface of the copper block. Finally, heat is removed on that surface which is called the spray cooling surface. The surface area is $14 \mathrm{~mm} \times 14 \mathrm{~mm}$. According to the simulation results as shown in Fig. 3, the temperature distribution along the neck of the copper block is a uniform gradient. Hence one-dimensional heat conduction is assumed and the heat flux can be calculated from the temperature measured.

There are six thermocouples are inserted in the neck of the copper block and three of them are a mirror image of the others. By this way, the error in heat flux measured can be reduced. According to Fourier's law for heat conduction, the heat flux density and the temperature of the cooled surface can be calculated by Eq. (1) and Eq. (2) respectively.

$$
\begin{gathered}
q=\frac{k \Delta T}{A \Delta x} \\
T_{S}=\left(T_{1}+T_{1 b}\right) / 2-\frac{\bar{q} X_{1-S} A}{k}
\end{gathered}
$$

In our experiment, the power input is set by the power control module. For one power input, there is a corresponding heat flux and surface temperature calculated.

\section{Green laser flow visualization technology}

HD video camera is used to observe the spray pattern as well as the flow pattern of the liquid film. However, the spray condition is too special to obtain ideal photographs only using ordinary camera technology. In order to study the evolution process of the liquid flow, green laser flow visualization technology is introduced in this experiment. Due to its advantage in highly imaging, the green laser flow visualization technology can be applied to many kinds of fluid mechanics experiments

The main flow visualization devices:

1) Semiconductor laser: the semiconductor laser is a double lumen Nd: YAG pulse laser. The maximum power is $5 \mathrm{~W}$ and the laser wavelength is $532 \mathrm{~nm}$. The single pulse energy is $200 \mathrm{mj}$ and the range of pulse is 6-8ns. The repetition frequency is $1-15 \mathrm{~Hz}$. Cylindrical lens is used to expand the original laser beam into a green sheet light with a certain angle.

2) HD video camera: because the liquid flow is instantaneous and dynamic, it is difficult for the ordinary camera to catch the entire flow process. The SONY HD 
video camera is used to record the whole evolution process about liquid flow.

\section{Flow visualization method}

The laser equipment is located behind the spray cooling setup and the cylindrical lens is fixed on the semiconductor laser in a certain angle, then the sheet laser is created and the observation area can be lightened ${ }^{[9]}$. Shading cloth is used to keep out light and make the camera shooting area a darkroom. Before the experiment, the angle and strength of the sheet laser is adjusted to make sure the green laser get to the ideal location. Afterwards, the liquid flow can be observed and recorded by the camera as shown in Fig. 4.

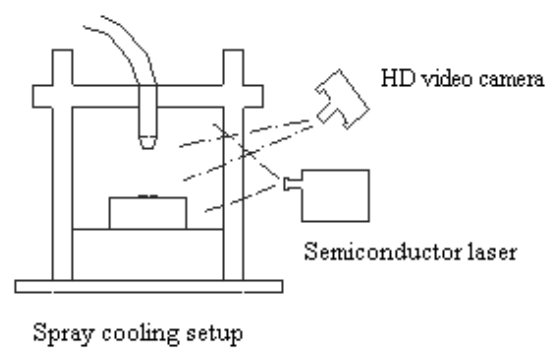

Figure 4. Sketch of flow visualization method

\section{THE HEAT TRANSFER SURFACE AND THE GUIDING SURFACE}

During the spray cooling process, liquid droplets are sprayed onto the heat transfer surface and then cool the surface ${ }^{[10 \sim 11]}$. Some of the droplets will change phase from liquid to vapor and the others will flow away from the guiding surface for recycling. Two different heat transfer surfaces are tested in this experiment as shown in Fig. 5 and Fig. 6, and they are flat surface and porous tunnel surface.

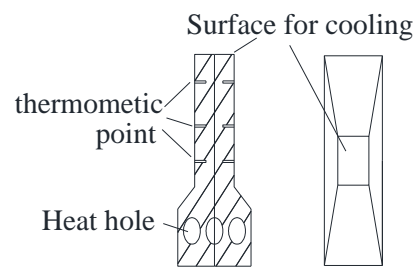

Figure 5. Flat surface

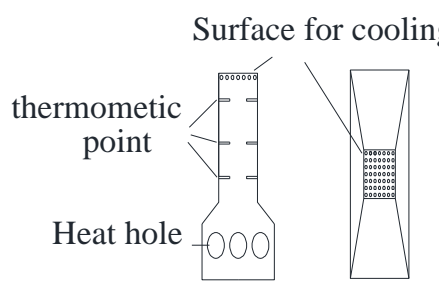

Figure 6. Porous tunnel surface

\section{THE COMPARISON OF THE SPRAY COOLING PERFORMANCE}

In this experiment, the height from the nozzle outlet to the heat transfer surface is $20.10 \mathrm{~mm}$ and the nozzle inlet pressure is $1.0 \mathrm{MPa}$. The water temperature is $23{ }^{\circ} \mathrm{C}$ and the heating power is $95.46 \mathrm{~W}$. The spray cooling performance is studied for the flat surface and the porous tunnel surface. The experimental results are shown in Fig. 7 and Fig. 8.

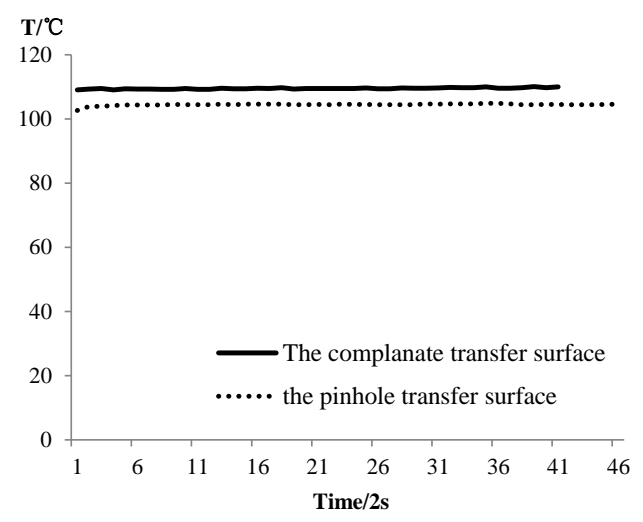

Figure 7. Comparison results about surface temperature

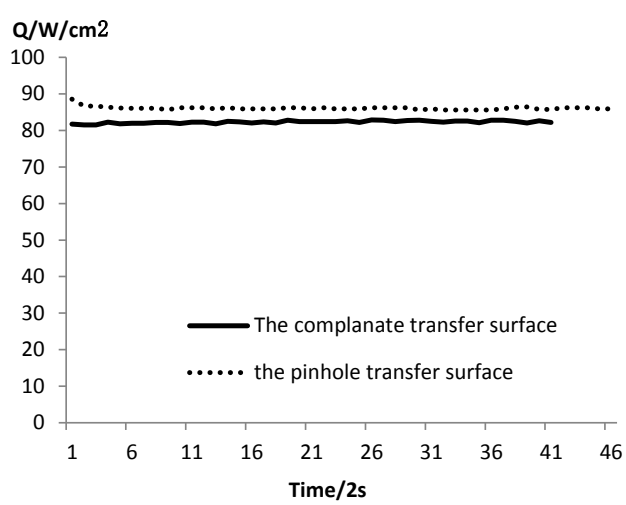

Figure 8. Comparision results about heat flux density

When the heating power is improved to $203.4 \mathrm{~W}$, the results about the temperature and the heat flux density are shown in Fig. 9 and Fig. 10.

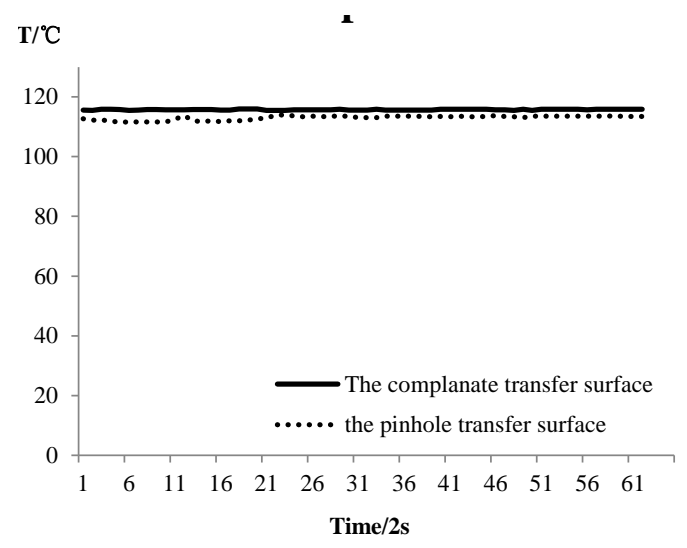

Figure 9. Comparison results about surface temperature 


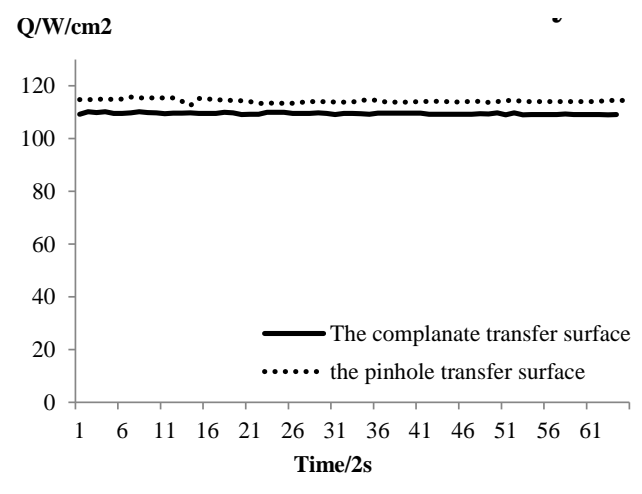

Figure 10. Comparision results about heat flux density

\section{ANALYSIS ABOUT EXPERIMENTAL RESULTS}

\section{A. Analysis about the experimental data}

According to Fig. 7 and 9, for a certain heating power, the temperature of the flat surface is lower than that of the porous tunnel surface. Besides, as the heating power is improved, the temperature difference between the two surfaces is decreased.

According to the Fig. 8 and 10, for the same heating power, the porous tunnel surface can achieve higher heat flux density compared to the flat surface.

\section{B. Liquid flow visualization results}

According to Fig. 11, in the two phase region, the cooling liquid changes into gas rapidly when it touches the heated surface and the flow of gas can be seen clearly.
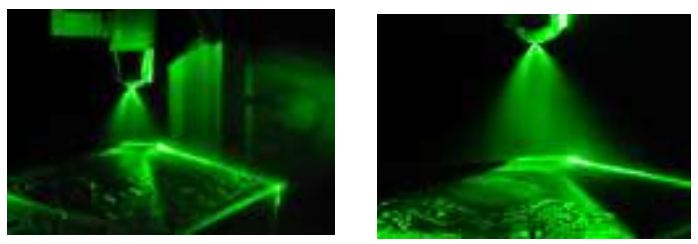

Figure 11. Liquid flow visualization results

\section{CONCLUSION}

In this paper, experimental method is used to research the heat transfer performance of spray cooling. A spray cooling system is set up to investigate the effect of installation angle on the cooling performance and green laser flow visualization technology is used to observe the liquid flow on the heater surface. Two types of cooling surfaces were studies experimentally. From the experimental results, we can draw the following conclusions:

Firstly, according to the comparision results about surface temperature as well as heat flux density, the porous tunnel surface has better heat transfer performance than flat surface.

Secondly, according to the liquid flow visualization results, it is observed that in the two phase region the liquid droplets change into gas the moment it touches the heat transfer surface.

\section{ACKNOWLEDGMENT}

The research is sponsored by the Aviation Science Foundation of China (2011ZC09004).

\section{REFERENCES}

[1] H. Baumann, P. Heinemeyer, and W.Staiger, "Optimized cooling system for high power semie conductor deviees," IEEE Transactionon Industrial Eleetronies, vol. 48(2), pp. 298-306, 2001.

[2] P. Miguel, C. Andre, M. Antonio. High-power electronics thermal management with intermittent multijet sprays, "Applied Thermal Engineering", vol. 37, pp. 293-301, 2012.

[3] Z. Zhang, P.X. Jiang, Y.T. Hu and J. Li, "Experimental Investigation of Continual- and Intermittent-Spray Cooling", Experimental Heat Transfer, vol. 26, pp. 453-469, 2013.

[4] W. Qu, and I. Mudawar, "Experimantal and numerieal study of the pressure drop and heat transfer in a single-phase micro-channel heat sink," Int.J.Heat Mass Transfer, vol. 45, pp. 2549-2565, 2002.

[5] E.A. Silk, E.L. Golliher, R.P. Selvam, "Spray cooling heat transfer: Technology overview and assessment of future challenges for micro-gravity application", Energy Conversion and Management, vol. 49(3), pp. 453-468, 2008.

[6] W. Jia, anf H. H. Qiu, "Experimental investigation of droplet dynamics and heat transfer in spray cooling," Experimental Thermal and Fluid Seience, vol. 27, pp. 829-838, 2003.

[7] R.P. Selvam, M. Sarkar, S. Sarkar, R. Ponnappan, and K.L. Yerkes, "Modeling thermal-boundary layer effect on liquid-vapour interface dynamics in spray cooling", Joural of Thermophysics and Heat Transfer, vol. 23(2), pp. 356-370, 2009.

[8] B. Horaeek, K.T. Esashi, and J. Kim, "Single Nozzle Spring cooling Heat Trangsfer Mechanisms," Iniemational Joumal of Heat and Mass Transfer, vol. 48, pp. 1425-1438, 2005.

[9] B. Horacek, J. Kim, and K. Kiger, "Spray cooling using multiple nozzles: visualization and wall heat transfer measurements," IEEE Transactions on Device and Materials Reliability, vol. 4(4), pp. 614-625, 2005

[10] M.K. Sung and I. Mudawar, "Single-phase and two-phase heat transfer characteristics of low temperature hybrid microchannel/micro-jet impingement cooling module", International Journal of Heat and Mass Transfer, vol. 51, pp. 3882-3895, 2008.

[11] J. R. Rvbicki and I. Mudawar, "Single-phase and two-phase cooling characteristics of upward facing and downward facing sprays", International Journal of Heat and Mass Transfer, vol. 49, pp. 5-16, 2006 